\title{
The Area of the Disk in Middle School Grade by GeoGebra.
}

\author{
https://doi.org/10.3991/ijet.v12.i11.6834 \\ David Lognoli \\ Scuola Secondaria di primo Grado "Giovanni da Verrazzano", Greve in Chianti, Italy \\ david.lognoli@gmail.com
}

\begin{abstract}
The visualisation and exploration of mathematical objects and concepts in multimedia environments can facilitate learning. Geometry is particularly involved with the growing use of software in teaching. GeoGebra is a dynamic geometry system that increases the level of knowledge and skills in mathematics. The use of GeoGebra permits one to complete and extend a teaching strategy based on manipulative activities.

The area of a disk in Middle School, i.e. Grades 7-8, offers the opportunity to use a large amount of geometry dynamic software. This paper proposes, as an example of combining in between manipulative activating and dynamic geometry software, the use of GeoGebra to permit the visualisation of three different approaches to the area of a disk. The first one is based on the limit of the area of inscribed regular polygons, the second one is based on dynamical morphing of the disk in a triangle and the third on sampling methods.
\end{abstract}

Keywords-GeoGebra, Teaching, Learning, Disk area, Dynamic geometry software, Middle School.

\section{Introduction}

Teaching activities and neurological studies suggest that the mathematic analphabetism mirrors a basic characteristic of our brain: its modularity, the compartmentalisation of mathematical knowledge into partially independent multiple modules. To become capable in math, it is essential to connect these modules by flexible links between them [1]. Students can benefit in different ways from technology integration into everyday teaching. The visualisation and exploration of mathematical objects and concepts in multimedia environments can facilitate understanding and new ways of learning [2]. In addition, the use of technology contributes to build an international community of teachers that can exchange their experience in an international contest, whereas traditional teaching is often confined to the national reality [3].

New learning opportunities are provided in technological environments, potentially engaging students of different mathematical skills and levels of understanding with mathematical tasks and activities [4]. Geometry is a domain of mathematical knowledge, which is particularly involved with the growing use of software in teaching because of the important role played by external representations, usually called 
figures, and the new ways of handling these representations that are made possible by software specifically designed for geometry [5]. On the other hand, simple drawings of mathematical objects and figures are not enough for the building of a comprehensive understanding of basic mathematical concepts. [6]. Consequently, it is important to support the use of dynamic geometry software by manipulative activities. This combined approach, a dynamic geometry software to complete and extend the manipulative activities, helps to avoid the fact that some students' work declines during classroom tasks. This approach is consistent with the general notion of mathematical understanding as one's growing competence to navigate through various representations of mathematical concepts is illustrated dynamically [6].

GeoGebra is a dynamic geometry system in which one works with points, vectors, segments, lines, and conic sections. On the other hand, equations and coordinates can be entered directly. These two views are characteristic of GeoGebra: An expression in the algebra window corresponds to an object in the geometry window and vice versa [7]. GeoGebra presents the main advantages in teaching and learning mathematics: easy teaching and easy learning, quick and correct grasping of the concept, and provision of an interactive learning environment [8]. The new teaching and learning method in mathematics, based on the use of the GeoGebra software in the teaching and learning process, causes much more increase in the level of knowledge and skills in mathematics than the traditional method used in this process [9]. The benefits of using GeoGebra software relate to independent and creative work, curiosity driving force, research opportunities, different science interactions, easy and better understanding of concepts, time benefit, wider and continually growing community of users, etc [9].

The area of disk learning is a suitable subject to show how starting by manipulative activities can be proposed the use of dynamic geometry software. The standard methodology used to explain the circle surface measure in junior secondary classrooms is not fully satisfying for the students and the teachers. The original Archimedes' proof [10] is out of range in these grades, and, for its complexity, isn't a real opportunity to improve competences and skills. More interesting are the proofs based on area rearrangement by Leonardo da Vinci [11] and Satō Moshun [12]. A traditional approach to these proofs is limited by the requirement to abstract the limit procedure without any visual support. Dart approximation is directly based on the Monte Carlo method and computing [13].

This paper proposes an approach to the circle area using three strategies based on dynamic geometry. These three approaches involve mathematic methods based on finite difference method and infinitesimal thinking. GeoGebra is a useful tool to link geometry, algebra, and calculus [14]. These methods can be considered too high for a junior secondary class but some qualitative studies show that when the students are engaged in high-level cognitive processes, they perform well produce good results, provided that the activeness is maintained [15]. So, because dynamic geometry is active, exploratory geometry carried out with interactive computer software [16], using dynamic geometry software as GeoGebra is a good idea, especially in a highlevel approach.

The educational method proposed in this paper is based on: 
- The visual approach to abstract contents [17];

- the simultaneous multiple skills advancement [18];

- the use of multiple student resources;

- the targets customisation to develop skills by giving value to different competences of students $[19,20]$.

\section{Target and objective.}

The target groups of these three activities are proposed for Middle School, i.e. Grades 7-8. It is assumed that the students have already known the measures of triangle and quadrilateral surfaces, the basic concepts of a circle, including center and radius and the distribution due by random events.

These activities are designed to be shown to the students by an interactive whiteboard or other interactive systems. Then the students work with the computer individually, or in pairs, or in small groups of 3-4. The objective of the individual or small group step is to consolidate their convictions and to experiment directly the situation obtained by parameters changing according to their curiosity.

Furthermore, the activities permit one to learn and gain knowledge to consolidate the area of the circle and to explore mathematic methods based on finite difference method and infinitesimal thinking.

It provides an opportunity for experiencing the process of exploring, conjecturing, verifying, justifying, and perhaps proving and becoming familiar with the visual approach to mathematic.

The activities are successfully experimented in a math's class.

\section{Area of the disk as the limit of the area of inscribed regular polygons.}

The first strategy proposed to indagate the area of the circle is based on inscribed regular polygons.

The developed GeoGebra code consists of a regular polygon inscribed in a circle. The circle, and consequently the polygon, can be rescaled on the graphic view of GeoGebra as shown on the left side in Fig. 1, Fig. 2 and Fig. 3. The center of the figure is connected to two consecutives vertices to show one of the congruent triangles which can share the polygon as shown on the right side in the figures. On the GeoGebra, the second graphic view shows the development of the polygon into triangles. The number of edges $(n)$ is controlled by a slide.

The students can infer that the polygon can be covered by a number of congruent triangles and that it is the same as the number of the edges of the polygon. Consequently, the measure of the surface of the inscribed polygon $\left(A_{\text {Polygon }}\right)$ is equal to the value obtained by multiplying the measure of the surfaces of one triangle $\left(A_{\text {triangle }}\right)$ by the number of the edges $(n)$. The measure of the surface of the triangle is obtained, as 
usual, by dividing the product of the triangle base and height $(h)$ by 2 . The triangle base is the same that polygon edge $(l)$.

Generally, the area of a polygon can be written as:

$$
A_{\text {Polygon }}=n \cdot A_{\text {Triangle }}=n \cdot \frac{l \cdot h}{2}=\frac{2 p \cdot h}{2}
$$

where $p$ is the half-perimeter.

It is important to emphasise that multiplying the edges number and edges length is obtained by the perimeter of the triangle, the measure of its border.

$$
n=3
$$
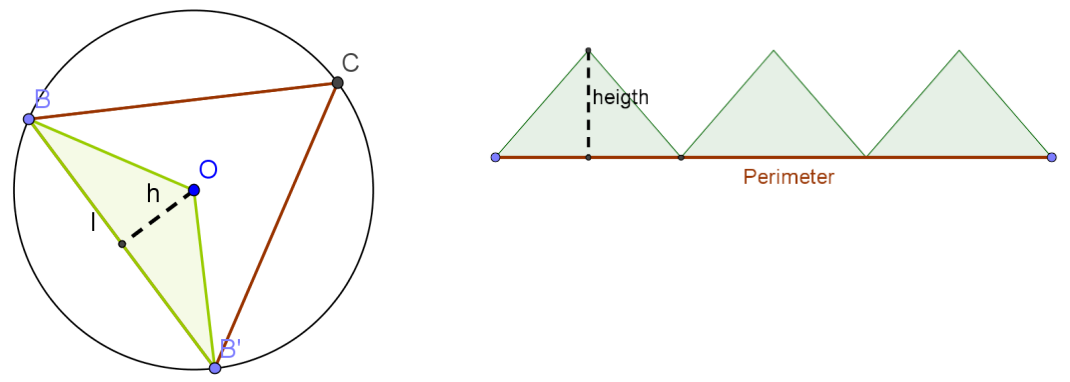

Fig. 1. On the left side, the GeoGebra graphic view with a 3-edges polygon inscribed; on the right side, the second GeoGebra graphic view shows the decomposition of the polygon in triangles. In the right size the axis aspect ratio (x:y) is 1:2.

In the cases of 3, Fig. 1, or 4 edges, the value of the height can be analytically retrieved from the equilateral triangle or square properties. In the equilateral triangle, it is enough to remember that the orthocenter divides the heights in $2 / 3$ and $1 / 3$.

Increasing the number of the edges makes the polygon fit better in the circle; its perimeter tends toward the circumference and the height of triangles to the radius. Fig. 2 shows the cases of an octagon $(n=8)$.

As shown for $n=25$ in Fig. 3, by moving the slide that controls the number of edges, the students can observe that:

- the inscribed polygon tends toward the circle;

- the triangle height tends toward the radius of the circle.

From the first observation, they can deduce that the length of the border of the polygon, the perimeter, tends toward the length of the border of the circle, the circumference $(c)$. Consequently, by (1) they can realise that:

$$
A_{\text {Circle }}=\frac{c \cdot r}{2}
$$




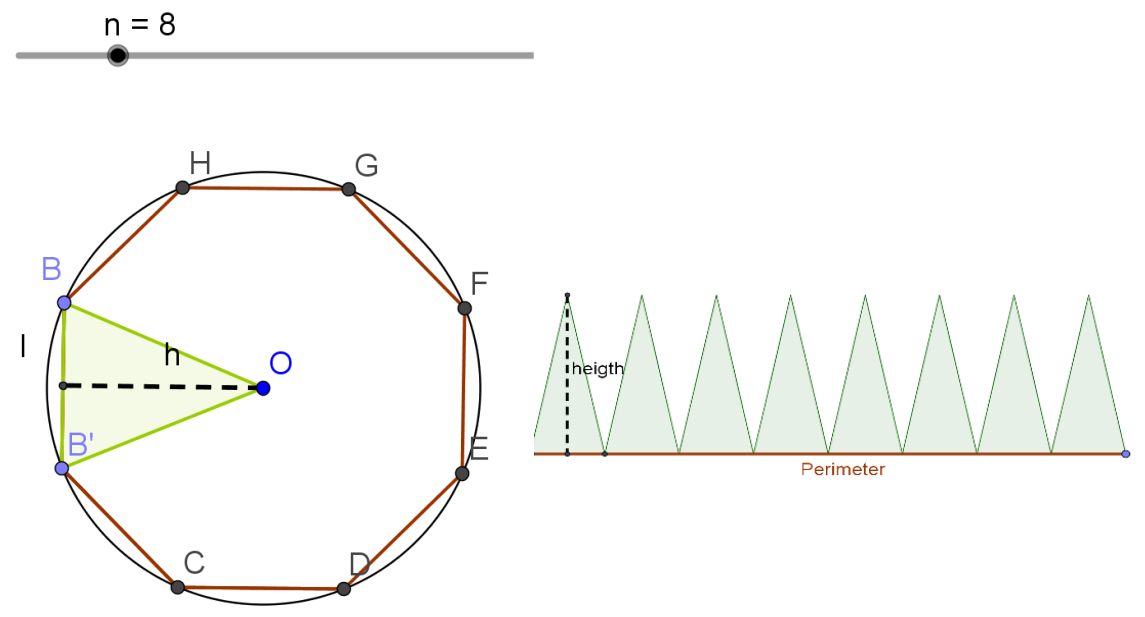

Fig. 2. On the left side, the GeoGebra graphic view with an 8-edges polygon inscribed; on the right side, the second GeoGebra graphic view shows the decomposition of the polygon in triangles. In the right size the axis aspect ratio (x:y) is 1:2.
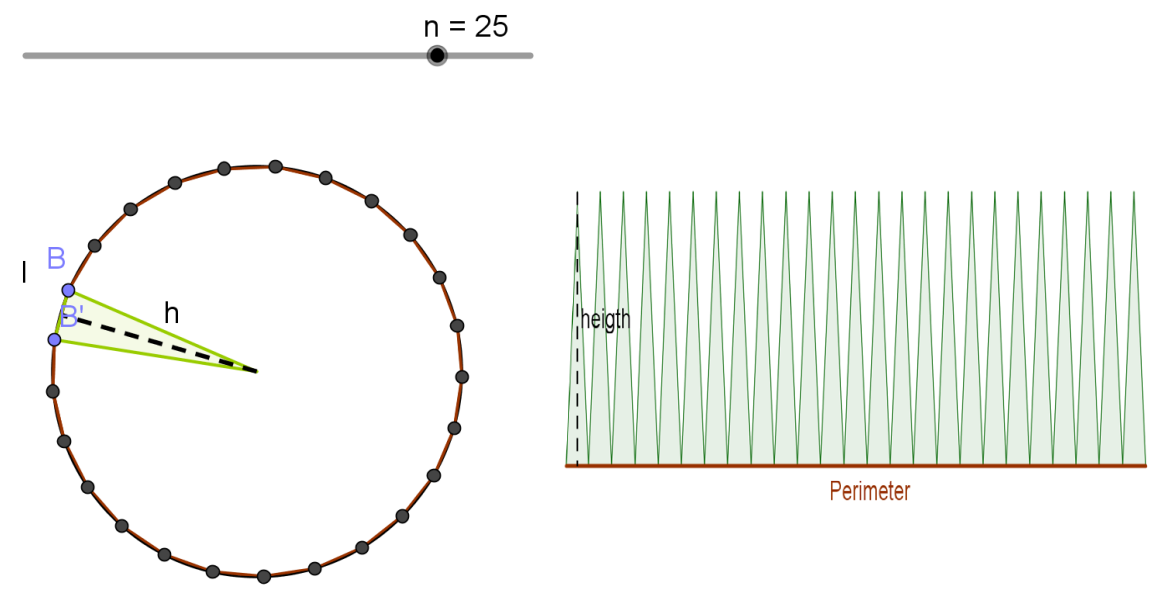

Fig. 3. On the left side, the GeoGebra graphic view with a 25-edges polygon inscribed; on the right side, the second GeoGebra graphic view shows the decomposition of the polygon in triangles. In the right size the axis aspect ratio (x:y) is 1:2. 
Substituting its expression as a function of the radius to the measures of the circumference (2) becomes:

$$
A_{\text {Circle }}=\frac{c \cdot r}{2}=\frac{2 \cdot \pi \cdot r \cdot r}{2}=\pi \cdot r^{2}
$$

\section{$4 \quad$ Area of the disk as the area of a triangle with the base length as the circumference and the height length as the radius.}

The second strategy proposes to indagate the area of the disk based on dynamical morphing of the disk in a triangle. The original idea was composed to divide the disk in several concentric annuluses, and then to cut and to open each of them as a "rectangle". The wider rectangle is set at the basis and the other ones are stacked over it to build a terraced triangle. As the number of annuluses increase, their height decreases and the final figure tends towards a triangle [21, 22]. An engaging story for young people, based on this idea, involves a licorice roll that is cut and stacked to build the triangle [23].

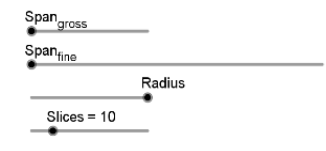

a)

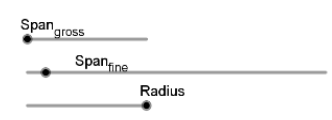

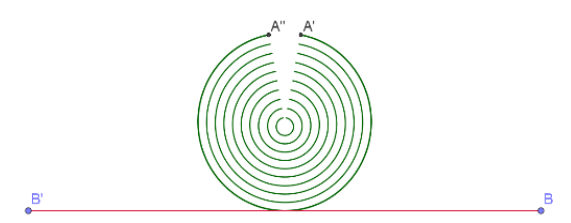

d)

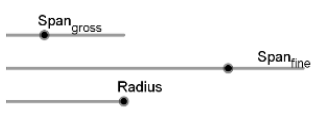

c)
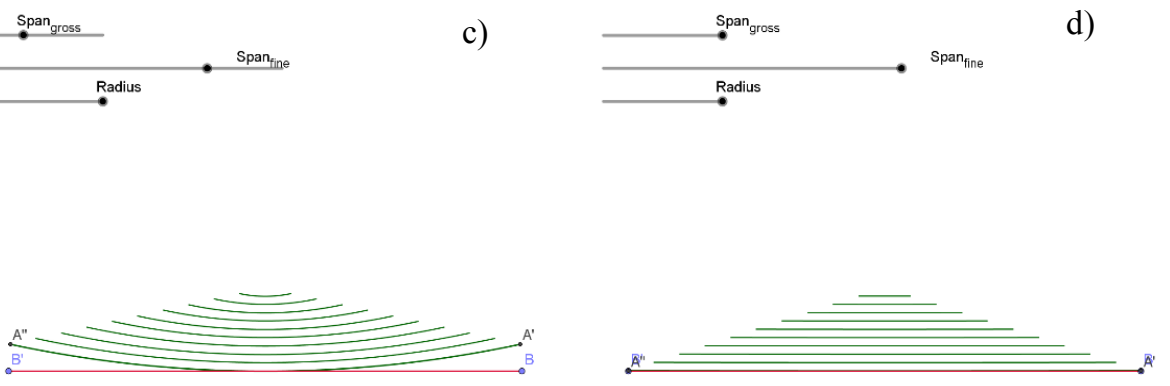

Fig. 4. GeoGebra graphic view of 10 concentric annuluses. (a) Span is zero, the annuluses are closed; (b) Span is low, the annuluses are opening; (c) Span is high, the annuluses are open; (d) Span reaches maximum value, the annuluses are turned into segments.

The developed GeoGebra code, Fig. 4, is composed in a circle that can be opened by two slides that drive the span, one slide for fine control and the other one for gross 
control. The code permits to set the circle radius and the number of annuluses, named "slices" in the control slide. There is a segment length as the circumference tangent to the circle.

The students, before taking on the area of the circle, worked on the length of the circumference with a real object and a twine. So, they knew that the circumference can be stretched in a segment of the same length. The class discussed about the significance of the cutting act and the differences between a closed set and an open one.

Fig. 4 shows the circle divided into 10 annuluses for four different values of span. By the slides of span, the control is continuous and the development can be shown to the class as a continuous transformation. It is important to observe that the length of segment $\mathrm{BB}^{\prime}$ is the same than the measure of circumference, and the center of the circle is fixed, so, the distance between it and BB' is the radius of the circle and the height of the triangle.

When increasing the slide that controls the number of annuluses, the circle can be filled by annuluses. In this way, moving the span slide control will make the circle turn into a triangle that has a base the length of the circumference and a height that is equal to the radius of the circle, Fig. 5. When moving back the slide, it is possible to turn the triangle into the original circle. Then, because the circle was transformed into a triangle with the same area and vice versa, the area of the circle can be obtained from the area of the triangle as shown in (3).
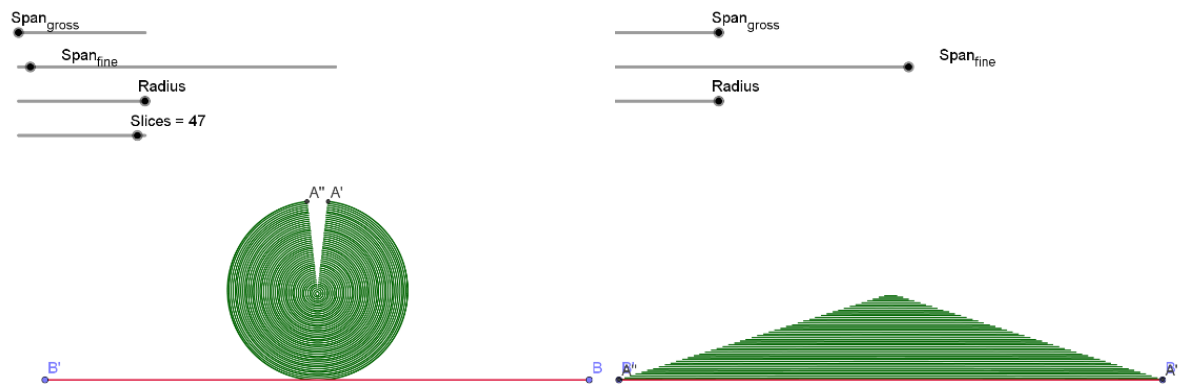

Fig. 5. GeoGebra graphic view of 47 concentric annuluses: on the left, the span is low, and consequently, the annuluses are opening; on the right, the span reaches maximum value; the annuluses are turned into segments and the circle is turned into a triangle.

\section{Area of the disk by sample method}

The third strategy proposes to indagate the area of the circle based on the random or uniform generation of points inside a square circumscribed on the circle. By this way, it is possible to retrieve the value of $\pi$, too [23]. 


\subsection{Finding the area of the disk using the Monte Carlo method}

The first idea is based on the Monte Carlo Method [24, 25]. To perform this activity, the class is required to assimilate the concept of uniform distribution in an interval and can calculate the distance between 2 points when the coordinates are known.

The developed GeoGebra code is contained in a square box with the inscribed circle. A slide drives the number of points randomly generated inside the square. If the distance between the center of the circle and the point, measured by the Pythagoras theorem, is equal or less than the radius measure, only then the point is part of the circle. If the random points are uniformly distributed, the ratio between the number of points that are inside the circle and those that are the ratio between the measure of the square surface and the measure of the circle surface:

$$
\text { ratio }=\frac{\text { Area }_{\text {circle }}}{\text { Area }_{\text {square }}}=\frac{\text { Points into the circle }}{\text { Points }}
$$

Being confident with the use of GeoGebra permits the class to be confident with the results obtained by GeoGebra, so, the code can be used to count the points inside the circle. The total number of points is controlled by the slide.

It is important to associate the points generated into the square as a random shot; the name of the GeoGebra command is "RandomPointIn" and it permits to stress this concept. The GeoGebra button that generates the points is named "shot" according to Fig. 6 and Fig. 7, which show the GeoGebra graphic view obtained with 176 and 3450 points, respectively. The code permits to control, by a slide, the radius of the circle too. The value of the circle radius is set to 1 in this example.

When moving the slide that controls the number of points, the student can observe that the value of the ratio tends towards a constant, and that the constant is 0.785 $(\pi / 4)$. When increasing the number of points, the precision of ratio value increases and can be added by decimal places to $\pi / 4$ value.

By the GeoGebra Spreadsheet View User Interface, the values of the number of points and of the ones inside the circle can be recorded. The recorded data are plotted on the second GeoGebra graphic view, Fig. 9 on the left, to show the convergence of the $\pi / 4$ value. Unfortunately, the convergence is slow. To fit the third decimal position of $\pi$, more than 100000 points are necessary.

The measure of the circle surface can be obtained by (4):

$$
\text { ratio }=\frac{\text { Area }_{\text {circle }}}{\text { Area }_{\text {square }}} \Rightarrow \text { Area }_{\text {circle }}=\text { ratio } \cdot \text { Area } a_{\text {square }}
$$

and by substituting the area of the square with the square of the edge that is two times the radius $r$ of the circle:

$$
\text { Area }_{\text {circle }}=\text { ratio } \cdot \text { Area }_{\text {square }}=\text { ratio } \cdot(2 \cdot r)^{2}=4 \cdot \text { ratio } \cdot r^{2}
$$

The successive step is naming the value of the 4-times ratio as $\pi$, so that the measure of the circle surface can be written as:

$$
\text { Area }_{\text {circle }}=\underbrace{4 \cdot \text { ratio }}_{\pi} \cdot r^{2}=\pi \cdot r^{2}
$$



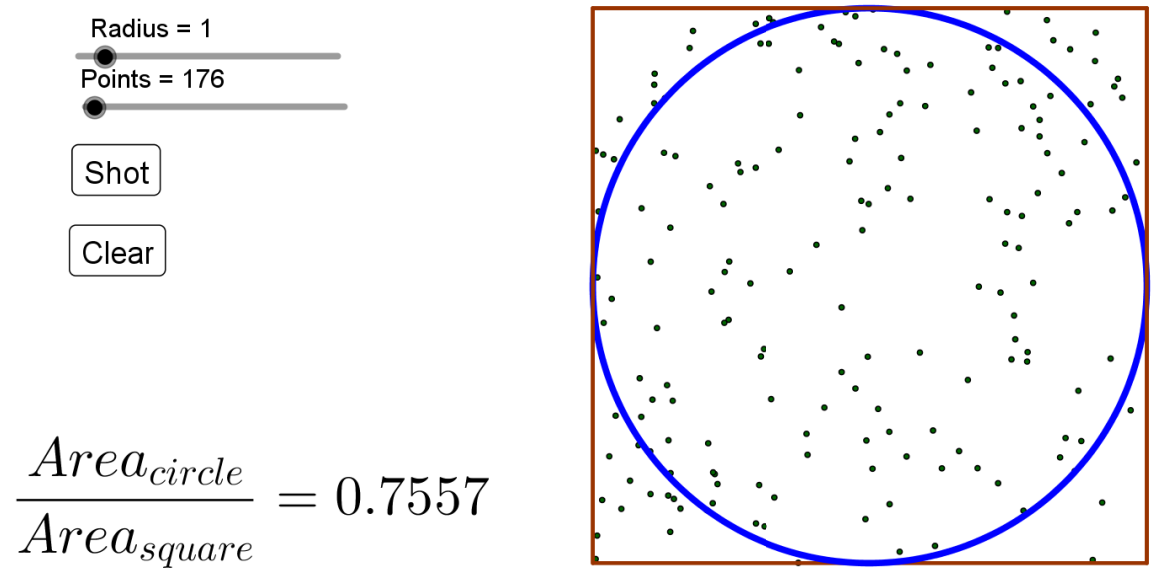

Fig. 6. GeoGebra graphic view obtained with 176 points. Some points are inside the circle, while others are outside. The ratio is shown on the graphic view.
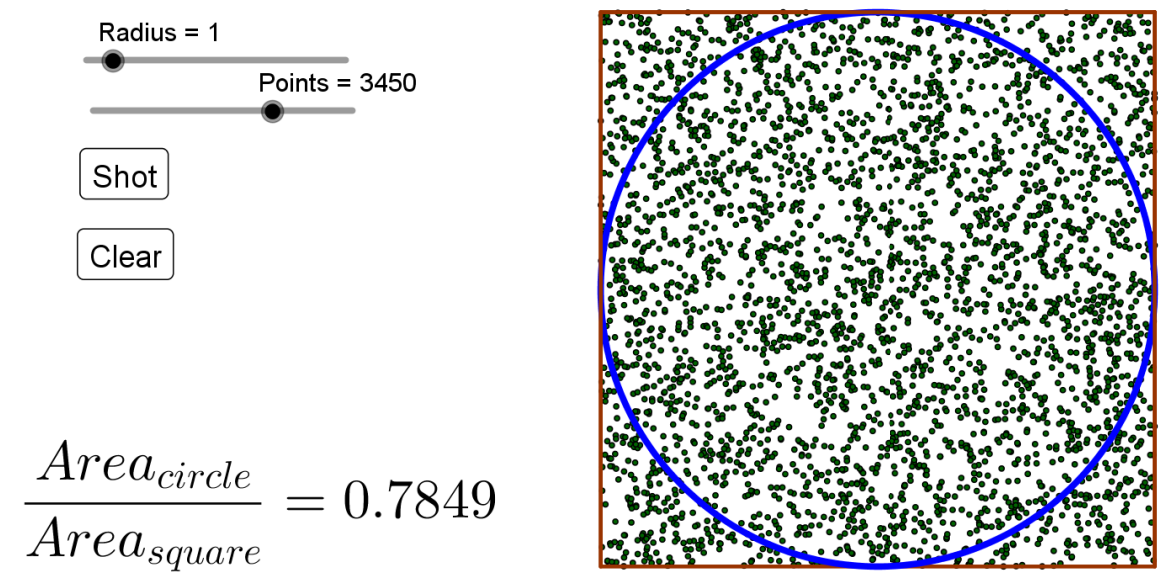

Fig. 7. GeoGebra graphic view obtained with 3450 points. Some points are inside the circle, while others are outside. The ratio is shown on the graphic view.

By this strategy, it is possible to find the $\pi$ value too. The accuracy of the retrieved value depends on the number of shot points.

\subsection{Area of the disk by uniform sampling method}

An interesting variation of the sampling method consists of the use of a regular distribution inside the random one. A diffuse use of Pick's rule [26, 27] in the junior 
secondary school made it usual to measure the area of a polygon arranged on a regular point grid.

The developed GeoGebra code, is contained in a square box with the inscribed circle. A slide drives the number of points generated inside the square along each of the two dimensions; each point appears as the vertex of a regular grid. If the distance between the center of the circle and the point, measured by the Pythagoras theorem is equal to or less than the radius measure, only then is the point a part of the circle.
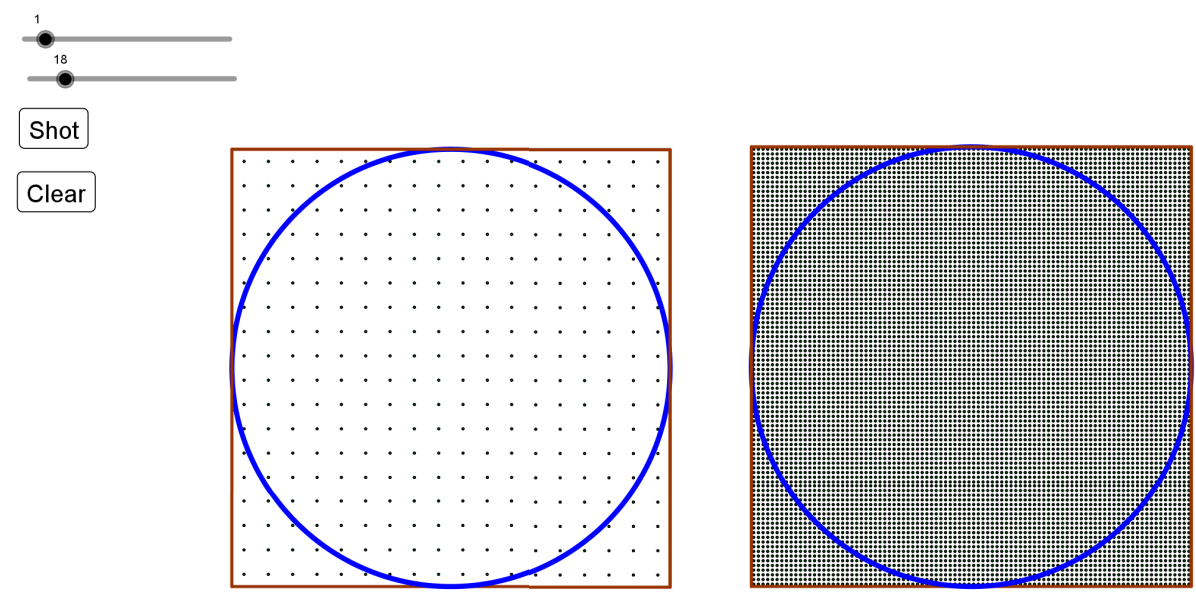

Fig. 8. GeoGebra graphic view obtained on the left with 324 points and the right with 9604 points. Some points are inside the circle, while others are outside.

The points are uniformly distributed. So, the ratio between the number of points that are inside the circle and those that are the ratio between the measure of the square surface and the measure of the circle surface. The ratio is given by (4). The total number of points is controlled by the slide. Fig. 8 shows the GeoGebra graphic view obtained with $18^{2}$, i.e. 328 , and $98^{2}$, i.e. 9604 , points. The code permits to control, by a slide, the radius of the circle too. The value of the circle radius is set to 1 in this example. When moving the slide that controls the number of points, the student can observe that the value of the ratio tends towards a constant, and that the constant is $0.785(\pi / 4)$. When increasing the number of points, the precision of the ratio value increases and can be added by decimal places to $\pi / 4$ value.

By the GeoGebra Spreadsheet View User Interface the values of the number of points and the ones inside the circle can be recorded. The recorded data are plotted on the second GeoGebra graphic view, Fig. 8 on the right, to show the convergence of $\pi / 4$ value.

The area of the disk can be obtained as shown in (7). By this strategy, it is possible to find the $\pi$ value too. The accuracy of the retrieved value depends on the density of points. 


\subsection{Convergence of the $\pi / 4$ value.}

Fig. 9 shows the comparison of convergence trends to $\pi / 4$ in the case on the left, of Monte Carlo method, and on the right, of the regular point grid. The graphs permit to discussion two very important argument:

- the approximation as the result of activity of measure;

- the difference between the convergence trends depending on the measurement method chosen.

The learning of approximation as the result of activity and not as abstract procedure is an important goal at the end of Middle School. A good knowledge of the approximation permits one to handle the real number set to reality application.

A comparison of the two trends puts in the evidence that regular sampling provides a faster convergence. From this experience, it is important to develop a discussion about the importance of organising and trying mathematical activities.
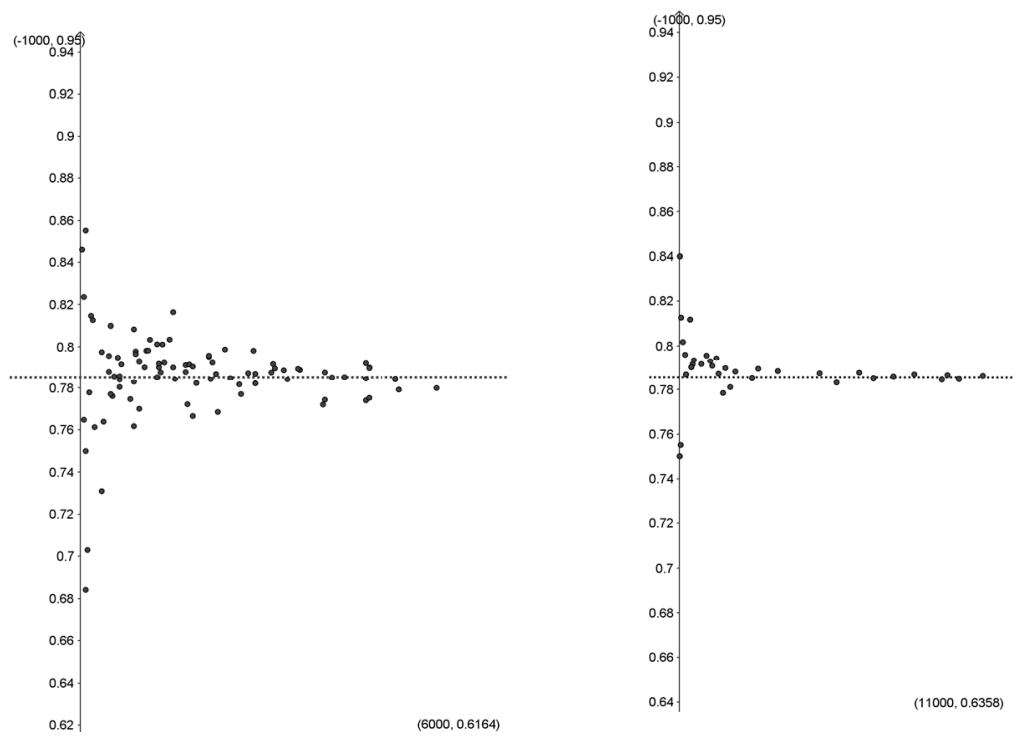

Fig. 9. GeoGebra second graphic views. On the left side is the ratio trend obtained by Monte Carlo method; on the right side is the ratio trend obtained by uniform distribution. Along the $\mathrm{x}$-axis is the number of points and along the $\mathrm{y}$-axis is the ratio. The horizontal line corresponds to the value of $\pi / 4$.

\section{Conclusion}

The combining of manipulative activities and modern technologies is a great opportunity to improve mathematical learning and teaching. Moving by a real class 
experience, the paper shows how GeoGebra can contribute to geometry teaching. Three strategies to teach disk area have been proposed, involving finite difference method and infinitesimal thinking, in a contest appropriated for Middle School grade.

\section{$7 \quad$ References}

[1] S. Dehaene, La Bosse des maths, Paris: Odile Jacob, 1997.

[2] J. Hohenwarter \& M. Hohenwarter, «Introducing Dynamic Mathematics Software,» Journal of Computers in Mathematics and Science Teaching, vol. 28, n. 2, pp. 135-146, 2008.

[3] J. Mousley, D. Lambdin \& Y. Koc, «Mathematics Teacher Education and Technology,» in Second International Handbook of Mathematics Education, Berlin, Srpinger, 2003, pp. 395-432.

[4] K. F. Hollebrands, «The Role of a Dynamic Software Program for Geometry in the Strategies High School Mathematics Students Employ,» Journal for Research in Mathematics Education, vol. 38, n. 2, pp. 164-192, 2007.

[5] C. Laborde, «The Computer as Part of the Learning Environment: The Case of Geometry,» in Keitel, Christine ;Ruthven, Kenneth, vol. 121, Berlin, Springer Berlin Heidelberg, 1993, pp. 48-67.

[6] D. Velichová, «Interactive Maths with GeoGebra,» International Journal of Emerging Technologies in Learning, vol. 6, n. Special Issue 1: "VU2009", pp. 31-35, 2011.

[7] C. Sangwin, «A Brief Review of GeoGebra: Dynamic Mathematics,» MSOR CONNECTIONS, vol. 7, n. 2, p. 36, 2007. https://doi.org/10.11120/msor.2007.07020036

[8] K. Pellumb, « GeoGebra: A Global Platform for Teaching and Learning Math Together and Using the Synergy of Mathematicians,» International Journal of Teaching and Case Studies, vol. 2, pp. 225-236, 2010. https://doi.org/10.1504/IJTCS.2010.033318

[9] A. Kllogjeri \& P. Kllogjeri, «Statistical Inferences Supporting the Hypothesis of Teaching with GeoGebra,» Open Access Library Journal, vol. 2, n. 1, pp. 1-10, 2015.

[10] Archimedes, The Works of Archimedes, Dover, 2002.

[11] P. Beckmann, A History of Pi, St. Martin's Griffin, 1976.

[12] D. E. Smith e Y. Mikami, A history of Japanese mathematics, Dover, 2004.

[13] J. Thijssen, Computational Physics, Cambridge University Press, 2006.

[14] J. Böhm, «inking Geometry, Algebra and Calculus with GeoGebra, ACDCA, DUG and Technical.,» University of Vienna, Vienna, 2008.

[15] M. Henningsen \& M. K. Stein, «Mathematical Tasks and Student Cognition: ClassroomBased Factors That Support and Inhibit High-Level Mathematical Thinking and Reasoning,» Journal for Research in Mathematics Education, vol. 28, n. 5, pp. 524-549, November 1997.

[16] J. King e D. Schattschneider, Geometry Turned On: Dynamic Software in Learning, Teaching, and Research, Cambridge: Cambridge University Press, 1997, p. ix.

[17] B. De Finetti, Il "saper vedere" in matematica, Loescher, 1967.

[18] L. Bazzini, «Il pensiero analogico nell'apprendimento della matematica» L'insegnamento della matematica e delle scienze, vol. 2, pp. 107-129, 1995.

[19] C. A. Tomlinson, The Differentiated Classroom: Responding to the Needs of All Learners, Alexandria: ASCD, 2014.

[20] M. Gentile, «Insegnare alla classe e personalizzare l'apprendimento,» L'Educatore, vol. 55 , n. 5, pp. 13-16, 2007.

[21] E. Castelnuovo, Didattica matematica, Firenze: La Nuova Italia, 1963.

[22] A. Cerasoli, I magnifici dieci, Firenze: Editoriale Scienza, 2011, pp. 146-155. 
[23] A. Cerasoli, Mister quadrato, Tireste: Editoriale Scienza, 2016, pp. 50-57.

[24] T. Van Hecke, «Fostering Understanding of Monte Carlo Simulations for Estimating $\pi$ Using Dynamic Geogebra Applets,» North American GeoGebra Journal, vol. 4, n. 1, pp. 9$15,2015$.

[25] J. M. Hammersley \& D. C. Handscomb, Monte Carlo Methods, London: Methuen, 1975.

[26] C. P. Robert, «Monte Carlo Methods,» in Wiley StatsRef: Statistics Reference Online, Wiley, 2016, pp. 1-13.

[27] C. R. Hirsch, «Pick's Rule,» The Mathematics Teacher, vol. 67, n. 5, pp. 431-443, 1974.

[28] G. Pick, " Geometrisches zur Zahlenlehre,» Sitzungsberichte des deutschen naturwissenschaftlich-medicinischen Vereines für Böhmen "Lotos" in Prag., vol. 19, pp. 311-319, 19.

\section{Author}

David Lognoli is a mathematics and science professor in Middle School. In the past, for a fifteen-year period, he worked as a researcher for the Institute of Applied Physics "Nello Carrara", Italian National Council of Researches. As a professor, he is involved in advanced teaching and learning methods combining manipulative activities and modern technologies. He would like to perform teaching as a laboratory experience in which the relationship between the teacher and students relates to reality science development.

Article submitted 04 March 2017. Published as resubmitted by the author 16 May 2017. 